\title{
Valor semiológico de la secreción mucosa genital de la recién nacida
}

\author{
Alberto Duarte Contreras*
}

RESUMEN: Toda recién nacida a término debe presentar en los primeros días de vida una secreción mucosa en el vestíbulo vaginal.

Esta secreción fisiológica no se encuentra:

1- En las recién nacidas prematuras.

2- En las recién nacidas a término con malformaciones congénitas que obstruyen la luz del canal genital.

3- En recién nacidas a término con atresia o con agenesia del cérvix uterino.

Estas malformaciones se pueden diagnosticar o al menos sospechar durante el período neonatal a partir de un "síntoma negativo", la falta de secreción cérvico vaginal en el introito vaginal.

Los métodos de diagnóstico son la clínica, la vaginoscopia y la ecografía.

El tratamiento es quirúrgico: en algunos casos se debe practicar de urgencia y en otros se pospone para la adolescencia.

PALABRAS CLAVES: Secreción vaginal en recién nacidas a término, vaginoscopía, malformaciones obstructivas. Síndrome de Rokitansky Küster Hauser, seudohermafroditismo masculino, seudohermafroditismo femenino.

SUMMARY: All newborn infant at term presents during the firts few days of life a mucous secretion at vaginal vestibule.

This physiologic secretion is absent:

1- In premature newborn infants.

2- In newborn infants at term with congenital malformations that obstruct the genital tract.

3- In newborn infant a term with atresia or with absence of cervix.

These malformations are diagnosed or, at least, are presumed during the neonatal period starting from "negative symptom": the absence of vaginal and cervical secretions at the vaginal introitus.

Diagnosis is based on the history, gynecologycal examination, vaginoscopy and echography.

The treatment is surgical: in some cases is urgent and in other may be postponed to adolescence.

KEY WORDS: Secretion vaginal in newborn infants, vaginoscopy, obstruct malformations. Rokitansky Küster Hauser. Syndrome. Pseudohermafroditism female and male.

Toda niña nacida a término presenta en el vestíbulo vulvar, durante los primeros días de vida, una secreción mucosa, fisiológica, abundante o escasa. Esta secreción no se aprecia en las recién nacidas prematuras; algunas niñas tampoco la presentan.

La presencia de esta secreción da certeza de integridad anatómica y permeabilidad del canal genital; cuando falta, se debe a obstrucción de la luz del canal por procesos embrionarios de neoformación o de agenesia. La falta de secreción es el único síntoma que existe para el diagnóstico precoz de dichas malformaciones $(4,10,14)$.

* Del Servicio de Ginecología de la Niña y la Adolescente del Hospital Erasmo Meoz. Cúcuta. Médico Honorario del Hospital Erasmo Meoz, Cúcuta.

\section{Fisiología cérvico vaginal en recién nacidas}

Fluhmann ha demostrado que en los dos últimos meses de la vida fetal las hormonas placentarias y maternas estimulan el epitelio cervical y vaginal y le causan cambios profundos. El endometrio fetal, en cambio, no reacciona a estas hormonas. Parece que los tejidos derivados del endodermo son los que mejor responden a las hormonas placentarias y maternas al final del embarazo (7).

El epitelio endocervical fetal desarrolla una clara actividad proliferativa y secretora que se aprecia nítidamente en la recién nacida. Las células de las glándulas endocervicales cambian de tejido columnar bajo a columnar alto y así, bien desarrolladas, acumulan y secretan glucógeno y mucina, la que cristaliza en forma de helecho.

El epitelio exocervical es la continuación del epitelio de la mucosa vaginal; se hipertrofia y se hace grueso, poliestratificado, escamoso y proliferativo. 
En los dos últimos meses de vida fetal tanto el epitelio exocervical como el epitelio del tercio superior o distal de la vagina, sufren una notable hipertrofia e hiperplasia, y asî se forman crestas altas y criptas profundas que constituyen intrincadas plegaduras transversales en la mucosa. Las plegaduras de la mucosa vaginal facilita su distensión. Estos pliegues no se encuentran en la mujer adulta $(8,14)$. (Figura 1).

Figura 1

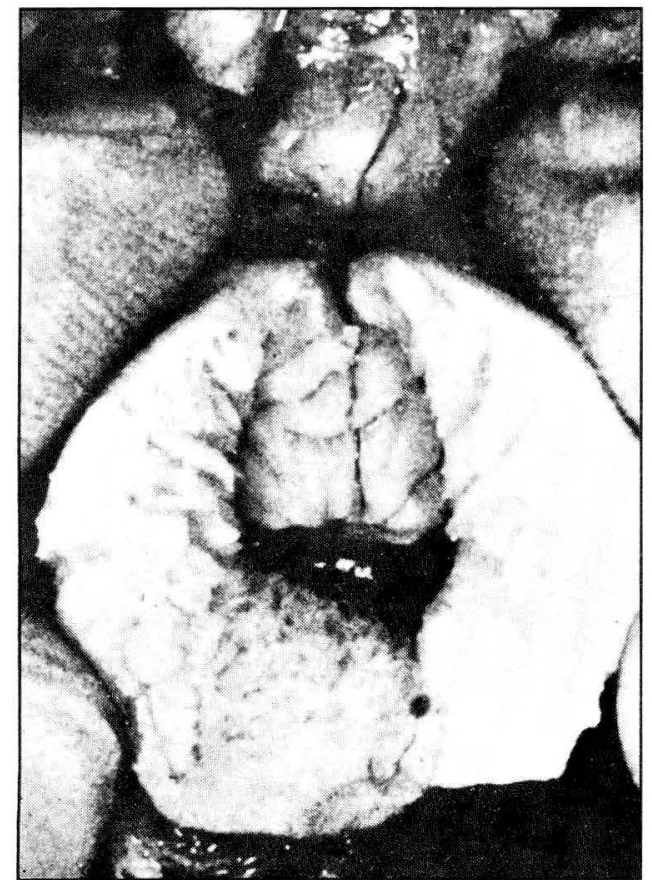

Muy visible el efecto del plegamiento en el exocérvix y en la porción superior de la vagina de la recién nacida. cantidad, o cierto grado de humedad en las paredes vaginales $(9,11,13)$

Todos estos cambios desaparecen pocos días después del nacimiento cuando ya no se encuentra en la circulación de las niñas influjo estrogénico materno o placentario $(1,7)$.

Las recién nacidas prematuras no presentan secreción cérvico vaginal en los primeros días de vida porque la acción sostenida de los estrógenos sobre cérvix y vagina sólo se efectúa de manera acentuada en los dos últimos meses del embarazo.

Cuando después de diez días persiste la secreción, se debe practicar examen bacteriológico, seguramente la secreción es bacteriana, específica, casi siempre gonocóccica.

\section{Investigación en la recién nacida}

En el examen ginecológico que por rutina se debe practicar a toda recién nacida es preciso investigar la presencia de secreción mucosa en el vestíbulo. Esta secreción, por lo general, es abundante y se aprecia a la inspección.

Con la sola presencia de secreción mucosa en la vulva de la recién nacida a término se puede diagnosticar con certeza desde el período neonatal la integridad del cérvix uterino y la permeabilidad del canal genital.

La falta de secreción mucosa nos obliga a iniciar un examen ginecológico detenido y metódico, examen que se inicia por el reconocimiento del himen. Para examinar el himen se flexionan y se abren las piernas; luego se separan los labios.

Si el himen es imperforado, sin hendidura, o tiene hendidura puntiforme o cribiforme (Figura 2), el hallazgo explica la falta de secreción: es éste el obstáculo que impide la salida de secreción. Se practicará de inmediato una himenotomía en la parte central. Con esta himenotomía queda permeabilizado el canal genital, evitando así la formación de un mucocolpos (4).

Figura 2

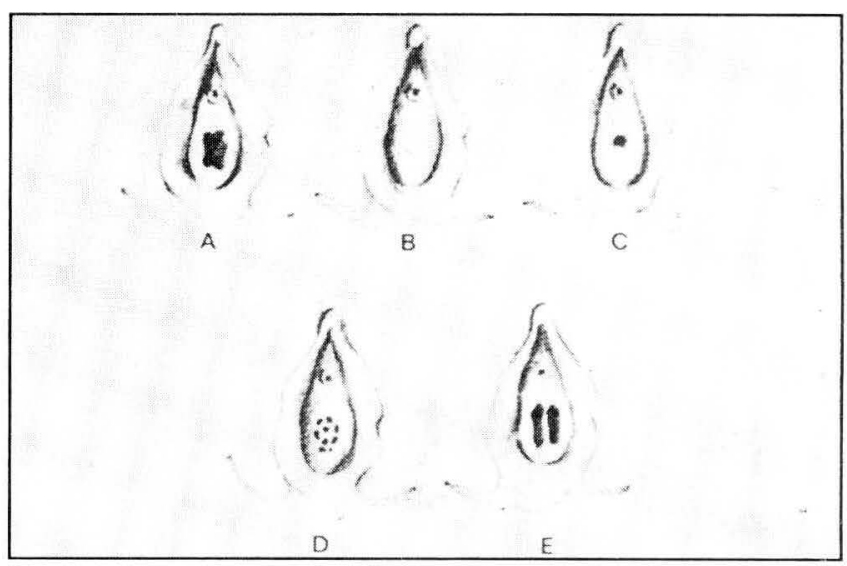

A-himen con hendidura normal; B- himen omperfordo, sin hendidura; C-himen con hendidura puntiforme: D- con hendidura cribiforme; E- con hendidura septada o bifenestrada.

Si el himen tiene hendidura central normal pero no se aprecia que por ella salga secreción, se hace una presión suave con el dedo índice sobre la horquilla a la entrada del vestíbulo (Figura 3 ). Con esta maniobra los bordes de la hendidura quedan separados y se visualiza la parte proximal de la mucosa vaginal, la que aparece de color rosado. Si con la presión ejercida sale secreción mucosa a través de la 
hendidura, así sea escasa, es señal de que hay cérvix normal que produce moco y de que no existe obstáculo alguno en la luz vaginal. Si con esta maniobra no se obtiene secreción, se practica un tacto rectal con el dedo meñique bien lubricada y se hace expresión suave de arriba a abajo por dos o tres veces (Figura 4). Con esta maniobra, si hay retención de secreción mucosa, ésta sale por le hendidura himeneal.

\section{Figura 3}

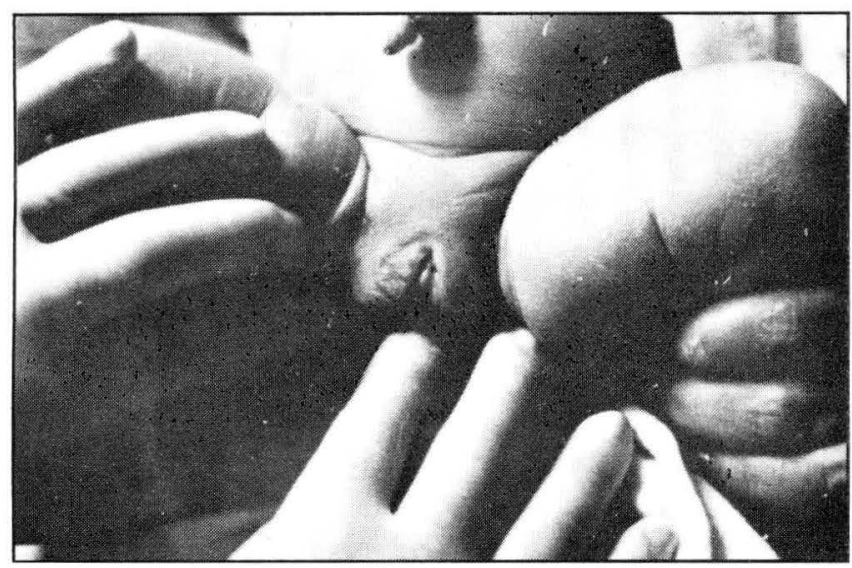

Manera de hacer una presión suave con el dedo índice sobre la horquilla a la entrada del vestíbulo.

Figura 4

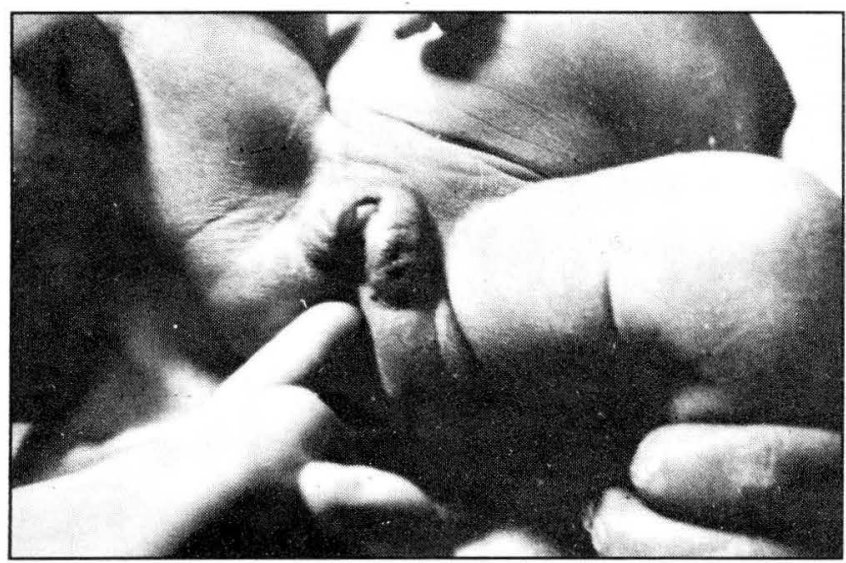

Se practica un tacto rectal con el dedo meñique bien lubricado y se hace expresión suave de arriba hacia abajo por dos a tres veces.

Si con ninguna de estas dos maniobras se obtiene secreción mucosa, hay un obstáculo en el canal vaginal, por encima del plano himeneal que impide su exteriorización (septo vaginal transverso, atresia o agenesia vaginal parcial o total), o el cérvix presenta un defecto anatómico (atresia o agenesia). En igual forma puede haber una agenesia vaginal, o un Síndrome de Rokitansky Küster o un Síndrome de Testículo Feminizante o Síndrome de insensibilidad a los andrógenos (10), diagnósticos que en igual forma se pueden plantear desde el período neonatal.

Es indispensable saber cuál es el obstáculo que obstruye el canal genital por encima del plano himeneal. Para ello es preciso buscar la profundidad o longitud de la vagina introduciendo por la hendidura himeneal un catéter de polietileno o una sonda de Nelatón No. 6 (Figura 5). En la recién nacida la vagina mide 4 centímetros. Si el catéter o la sonda solamente 2 a 4 milímetros es porque existe una obstrucción en el tercio inferior o proximal de la vagina, obstrucción que no puede ser otra cosa que un tabique transversal o una atresia vaginal.

\section{Figura 5}

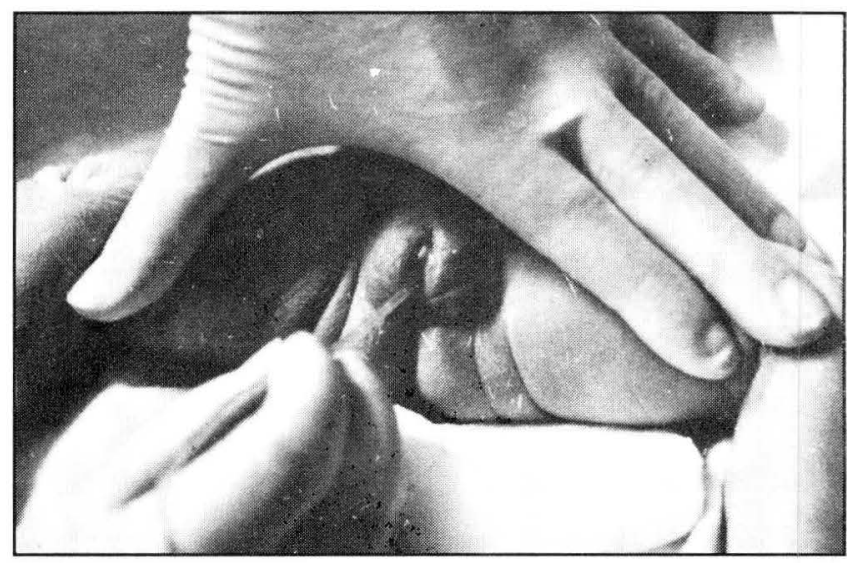

Para conocer la longitud de la vagina se introduce por la hendidura himeneal un catéter de polietileno o una sonda de Nelatón No. 6.

Cuando el tabique está muy cerca del himen, de 1 a 3 milímetros, se puede observar, con buena luz, a través de la hendidura himeneal, al tensionar los labios hacia afuera. Cuando está a más de 3 milímetros es preciso realizar una endoscopia vaginal empleando un vaginoscopio o, en su defecto, un otoscopio que tenga una buena fuente de luz. El tabique se ve nítido como una membrana nacarada. Para confirmar el diagnóstico se intenta traspasar el obstáculo con una aguja empatada a una jeringa: si se logra esta maniobra con facilidad es señal inequívoca de que hay un tabique. El espesor del tabique en promedio es de un milímetro. Por esta aguja se pueden inyectar 2 a 3 centímetros de medio de contraste para tomar una genitografía. Confirmado el diagnóstico de tabique vaginal transverso, se hace de inmediato, a través del vaginoscopio o del otoscopio, una abertura o ventana amplia en la parte central del tabique con un bisturí en bayoneta, teniendo el cuidado de no lastimar ni la uretra ni el recto para lo cual se pasa previamente una sonda uretral y se introduce el dedo meñique en el recto. Al abrir esta ventana queda permeable el canal genital. Se informa a los padres que cuando la adolescente vaya a iniciar vida sexual, se le debe practicar una resección completa del tabique. El hallazgo de tabique transversal es más frecuente que el de himen imperforado y que todas las demás malformaciones del canal.

Cuando el obstáculo del tercio inferior de la vagina no da a la vaginoscopia, la sensación de una membrana plana, vertical, nacarada, sino el de una cúpula o embudo tapizado por un tejido rosado similar al que cubre la pared vaginal proximal, se trata de una atresia vaginal. En este caso es imposible traspasar el obstáculo con una aguja dedo el espesor del tejido que siempre es de 3 o más milímetros. Se practica entonces con toda suavidad un tacto rectal y, ya con alguna experiencia, se puede palpar un tejido duro, de mayor o menor longitud según el tamaño de la atresia, que reemplaza al tejido vaginal suave normal. La ecografía nos informa la longitud de la porción distal de la vagina sana 
(14). El tejido atrésico se extirpa en la primera infancia solamente cuando está ocasionando un mucocolpos. Para extirparlo es indispensable conocer su longitud. Para ello se introduce una sonda por la hendidura himeneal y se mide la longitud de la porción vaginal proximal; por medio de la ecografía se mide la porción distal sana de la vagina; así, recordando que la longitud normal de la vagina en la recién nacida y a principios de la primera infancia es de 4 centímetros, y conociendo la longitud de las dos porciones de vagina sana, podemos conocer el tamaño del tejido atrésico. Cuando no se forma mucocolpos el tejido atrésico se extirpa en la adolescencia, cuando aparezca la criptomenorrea. La sola simple presunción de atresia obliga al médico informar a la familia para que en la adolescencia se tenga presente esta posibilidad como causante de criptomenorrea.

Cuando la atresia vaginal toma los dos tercios superiores de la vagina, o sea es completa, se acompaña en la mayoría de los casos de atresia o de marcada hipoplasia uterina. En este caso nunca se presenta mucocolpos en la infancia ni criptomenorrea en la adolescencia. Se informa a los padres la necesidad de hacer una neovagina en la adolescencia a más del futuro gineco obstétrico de esta niña.

En la recién nacida el útero mide entre 2.5 y 3.5 centímetros de longitud y, a pesar de la experiencia, es difícil localizarlo con el tacto rectal. La ecografía es el método sencillo y seguro para hacer el diagnóstico de presencia de útero, de hipoplasia o de agenesia uterina desde el período neonatal.

Cuando el himen tiene hendidura normal y la profundidad de la vagina es normal, con presencia de útero de tamaño normal a la ecografía, pero no se aprecia secreción mucosa ni se puede demostrar con las maniobras descritas anteriormente, se debe pensar en un defecto anatómico del órgano productor del moco: atresia o agenesia del cérvix. Este diagnóstico es de presunción y sólo se puede confirmar en la adolescencia si se presenta un hematometra. Se sabe entonces que hay útero funcionante. Por la vaginoscopia, la colposcopia, la citología funcional y a veces por la biopsia, se confirma el diagnóstico de la anomalía cervical. Es ésta una eventualidad muy rara.

En algunas recién nacidas no se encuentra himen y en su reemplazo se palpa un tejido irregular y plizado que recubre la depresión donde debería encontrarse el introito vaginal. De inmediato el médico examinador sospecha una agenesia vaginal. Al tacto rectal no se palpa la vagina, ni el cordón fibroso que se aprecia entre la uretra y el recto en los casos de atresia vaginal, sino un tejido muy blando, de relleno. La ecografía confirma la ausencia de vagina. Su tratamiento, la neovagina, se realiza cuando la adolescente desee iniciar vida sexual.

La agenesia de vagina se acompaña algunas veces de agenesia uterina y en otras el útero está reducido a dos cuernos rudimentarios no tunelizados, sólidos, visibles a la ecografía: es el Síndrome de Rokitansky-Küster-Hauser, Síndrome que se puede diagnosticar o al menos presumir desde el período neonatal. En este síndrome los ovarios y las trompas están presentes y son normales pero no se palpan al tacto rectal. Los ovarios se visualizan al estudio ecográfico, no así las trompas (14).

En otras recién nacidas ni se encuentra el himen ni se observa tejido plizado en el sitio donde debería estar el introito vaginal, y en su lugar se visualiza una fosita que remeda una vagina muy corta, de 2 a 3 milímetros de profundidad, en forma de fondo de saco. Al practicar el examen para investigar presencia de vagina se constata por tacto rectal y por ecografía que hay agenesia vaginal.
Además la ecografía muestra ausencia de útero y de ovarios. Con frecuencia estas recién nacidas tienen una o dos hernias inguinales, de contenido duro, que no reducen. $\mathrm{La}$ cromatina sexual es negativa y el cariotipo XY. Se sospecha de inmediato un seudohermafroditismo masculino. Se informa a los padres que más tarde se debe constatar el problema del seudohermafroditismo. La neovagina se hará cuando la adolescente desee iniciar su vida sexual. Este síndrome se encuentra entre 1 por 20.000 y 1 por 50.000 varones (6).

Un reducido número de recién nacidas con agenesia vaginal tienen órganos genitales internos normales (3).

Las niñas con seudohermafroditismo femenino no tienen vagina y ésta se encuentra reemplazada por un seno urogenital estrecho. Cuando el seno urogenital es muy estrecho se forma mucocolpos (Figura 6) y es preciso ampliarlo; entonces el tratamiento quirúrgico se debe practicar de inmediato. Cuando no hay mucocolpos, el tratamiento quirúrgico se realizará en el tiempo oportuno (14).

\section{Figura 6}

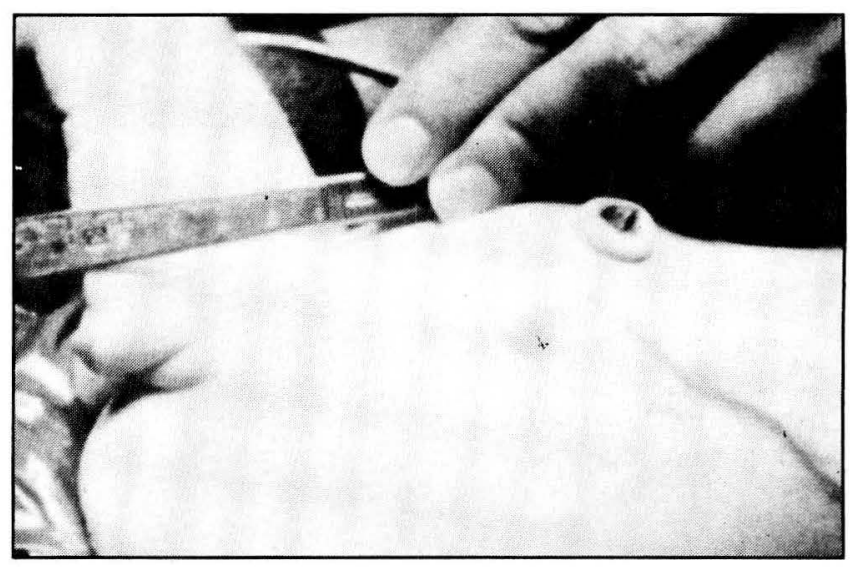

Se observa una masa abdominal entre el ombligo y el pubis en una niña de tres meses con seudohermafroditismo femenino y marcada estrechez del seno urogenital.

En algunas niñas a quienes no se ha practicado examen ginecológico en los primeros días de vida, se observa a comienzos de la primera infancia, una masa abdominal baja. Al tacto rectal y a la palpación rectoabdominal se ecuentra la vagina llena por un contenido blando, renitente, a tensión, que se continúa con la masa encontrada en el bajo abdomen. Es un hidrocolpos, llamado también mucocolpos que se forma por acúmulo de abundante secreción cérvico vaginal que no se ha exteriorizado a causa de un obstáculo en el tercio inferior o tercio medio de la vagina: himen imperforado, himen con hendidura puntiforme o cribiforme, tabique vaginal transverso o atresia vaginal parcial. Esta masa se visualiza por ecografía o por ginecografía cuando se inyecta medio de contraste a través del himen o del tabique. El tratamiento es quirúrgico, de urgencia, por vía vaginal, haciendo himenotomía o abriendo un ojal amplio en la parte central del tabique o retirando la porción atrésica de la vagina (13-15).

El hidrocolpos se puede encontrar también en seudohermafroditas femeninas cuando el seno urogenital es tan estrecho que no permite el drenaje de una secreción cérvico vaginal abundante (14). 


\section{Conducta}

El tratamiento de las malformaciones congénitas del conducto genital de las recién nacidas es quirúrgico. Se practica de inmediato en los casos de himen imperforado, himen con hendidura puntiforme o cribiforme, haya o no mucocolpos. El tabique vaginal y la atresia parcial de vagina se operan a principio de la primera infancia cuando hay mucocolpos. Cuando no hay mucocolpos se operan al iniciarse la adolescencia en presencia de la criptomenorrea. La agenesia vaginal se opera cuando la adolescente desee iniciar vida sexual.

Desafortunadamente la falta de examen ginecológico rutinario y metódico a la recién nacida y el hecho de haber un buen número de recién nacidas prematuras, hacen que estas alteraciones se descubran solamente en épocas posteriores, infancia o adolescencia, cuando se presentan complicaciones: hidrocolpos al comienzo de la primera infancia, hematocolpos, hematometra y hematosálpinx al iniciarse la pubertad $(2,5)$.

Lo ideal sería hacer un diagnóstico o al menos presuntivo en el período neonatal, el que se podría confirmar o no en la segunda infancia o al inicio de la pubertad. Este hecho nos habla del importantísimo papel preventivo que debe cumplir la ginecología infantil en el diagnóstico y tratamiento precoz de un buen número de padecimientos del aparato reproductivo.

Cuando la vulva se encuentra muy pulposa como respuesta del tejido genital a la acción estimulante estrogénica de finales del embarazo, el examen ginecológico se hace difícil y aún imposible. Entonces se pospone el examen para cuando haya cesado la acción hormonal, unos veinte días en promedio, o a más tardar en la segunda infancia.

Casi siempre las malformaciones congénitas del canal genital se acompañan de malformaciones de otros sistemas, en especial del tracto urinario (30\%); por ello se debe practicar siempre estudio radiológico de vías urinarias.

El diagnóstico comprobado o la presunción diagnóstica de estas malformaciones nunca se debe ocultar a la familia. Es indispensable dar un detallado informe de la malformación existente, del futuro ginecológico y obstétrico de la niña y, en muchos casos, a su debido tiempo, suministrar un eficiente soporte sicológico a la adolescente y a los padres.

\section{BIBLIOGRAFIA}

1. Dewhurst CJ. Endocrinología, anatomía y fisiología. En: Ginecología Pediátrica. Ed. Alhambra, S.A. Madrid. 1964; 1.

2. Dewhurst CJ. Obstrucción de vías genitales en niñas y adolescentes. Clin. Ped. N. Am. 1981; 331.

3. Dewhurst CJ. A simplified approach to management of congenital absence of the vagina and uterus. Pediatric and Adolescent Gynecol. 1984; 2: 24.

4. Duarte-Contreras A. Malformaciones congénitas de los órganos genitales. En: Ginecología de la Niña y de la Adolescente. Salvat Ed. Colombiana, S.A. Bogotá. 1988; 53.

5. Duarte-Contreras A. Criptomenorrea. Rev. Col. Obstet. Ginecol. 1989; 40: 313.

6. Duarte-Contreras A. Seudohermafroditismo masculino. En: Ginecología de la Niña y de la Adolescente. Salvat Ed. Colombiana, S.A. Bogotá. 1988; 180.

7. Fluhmann C. The nature and development of the so-called glands of the cervix uteri. Am. J. Obstet. Gynecol. 1957; 74: 753.
8. Huffman JW., Dewhurst CJ. and Capraro VJ. Anatomy and Physiology. En: The Gynecology of Childhood and Adolescence. W.B. Saunders Company, Philadelphia. 1981; 24.

9. McDonald C. Unusual forms of gynatresia. J. Obstet. Gynecol. Br. Emp. 1960; 67: 848.

10. Rey-Stocker I. La Ginecología en la niña. Anales Nestlé 1976; 126: 3.

11. Rey-Stocker I. Les infections des organes génitaux chez le nouveau-né, l'enfant et l'adolescente. Rev. Med. Suisse Rom. 1977; 97: 295.

12. Sannicandro G. Considerations sur l'anatomie microscopique de la muqueuse vaginale du foetus, du nouveau-né et de la fillette. VIII Cong. Franc. de Gynecol. 1939; 185.

13. Sen N. Hydrocolpos. Lancet. 1949; 2: 991.

14. Spence JEH., Wenning JB. and Martin D. Assessment and imaging of the pediatric and adolescent gynecologic patient. Adolesc. Pediatr. Gynecol. 1988; 1: 164.

15. Wilson D. Ultrasound diagnosis of hydrocolpos. Radiology 1978; 128: 451. 